\title{
Transesophageal echocardiography images of pulmonary artery compression by benign follicular lymphoid hyperplasia
}

\author{
Rob C. Tanzola, MD • Jason Erb, MD • \\ Ryan Endersby, MD • Brian Milne, MD
}

Received: 16 June 2010/ Accepted: 29 July 2010/Published online: 12 August 2010

(c) Canadian Anesthesiologists' Society 2010

\section{To the Editor,}

Whereas transesophageal echocardiography (TEE) has proven its utility in the cardiac surgical setting, its role in non-cardiac surgery is emerging, but less established. ${ }^{1} \mathrm{We}$ present a case in which TEE during mediastinoscopy was shown to be useful in detecting the degree of dynamic compression of the pulmonary artery and left atrium by benign follicular lymphoid hyperplasia. Consent for publication was obtained from the patient.

A previously healthy 32-yr-old woman presented to the emergency room with complaints of fatigue and chest pain. Pulmonary embolus was a consideration, so a computerized tomography (CT) scan of her chest was performed. The CT image was negative for a pulmonary embolus, but it did reveal a $6-\mathrm{cm}$ subcarinal soft tissue mass causing narrowing of the bronchus intermedius and exerting mass effect on the right pulmonary artery (PA) and left atrium (LA). Notably, despite her fatigue, the patient had no respiratory or postural symptoms, and her exercise tolerance remained greater than four metabolic equivalents. She presented to the operating room for a mediastinoscopy and biopsy for tissue diagnosis, as lymphoma was strongly suspected. Given the mass effect on the PA and LA, there was concern for hemodynamic instability with induction, positive pressure ventilation, and muscle relaxation. After insertion of an arterial line, the patient was induced with

Electronic supplementary material The online version of this article (doi:10.1007/s12630-010-9368-3) contains supplementary material, which is available to authorized users.

R. C. Tanzola, MD ( $₫)$ · J. Erb, MD · R. Endersby, MD .

B. Milne, MD

Kingston General Hospital, Queen's University,

Kingston, ON, Canada

e-mail: tanzolar@kgh.kari.net the following short-acting agents: midazolam $2 \mathrm{mg} i v$, followed by titrated doses of remifentanil (to a total of $1 \mu \mathrm{g} \cdot \mathrm{kg}^{-1} \mathrm{iv}$ ) and propofol (to a total of $2 \mathrm{mg} \cdot \mathrm{kg}^{-1} \mathrm{iv}$ ), with the initial goal being preservation of cardiovascular stability with maintenance of spontaneous ventilation. Ventilation was then taken over gradually with bag-mask to ensure that there was no hemodynamic instability once positive pressure ventilation was introduced. The patient's trachea was then intubated with an endotracheal tube and a TEE probe was placed. Only after the TEE provided reassuring images was the patient paralyzed with rocuronium $0.4 \mathrm{mg} \cdot \mathrm{kg}^{-1} i v$.

The TEE imaging revealed a large mass located posterior and extrinsic to the right PA that caused nearcomplete occlusion of the right PA (Figs 1 and 2, Videos 1 and 2). The left PA was free of clinically significant involvement, and there was minimal compression of the LA. A small pericardial effusion and a hyperdynamic left ventricle were also noted. No significant valvular abnormalities were present. Right ventricular systolic pressure, an estimate of PA systolic pressure, could not be calculated due to an inadequate tricuspid regurgitation Doppler signal. The patient remained stable throughout the procedure, and she was transferred to the postanesthesia care unit after tracheal extubation in the operating room. The pathology report indicated that the mass was follicular lymphoid hyperplasia with no evidence of malignancy. An inflammatory process was suspected, and this was supported by a repeat CT scan three months later that revealed complete resolution of the mass.

The goals of traditional management of mediastinal masses include the maintenance of spontaneous ventilation and the avoidance of muscle relaxants. ${ }^{2}$ Although this was the initial approach in this case, the patient was eventually paralyzed and her lungs were mechanically ventilated for 


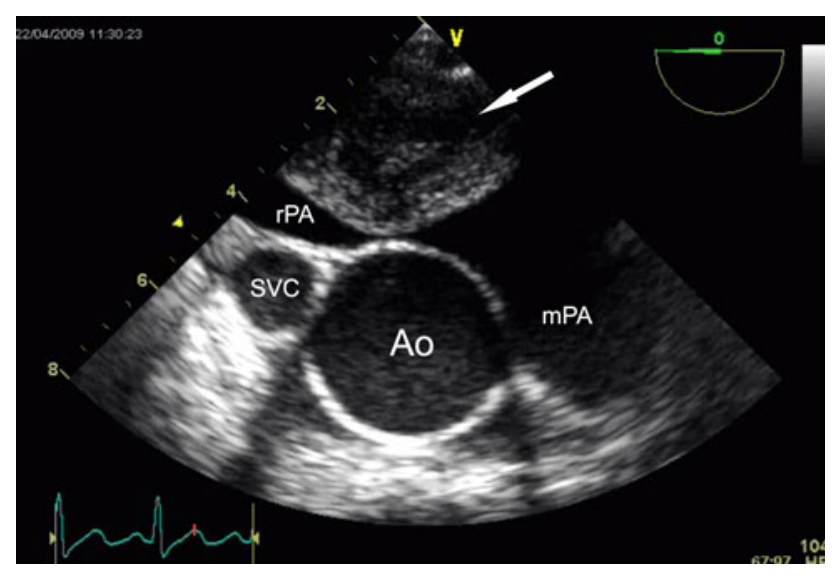

Fig. 1 A transesophageal echocardiogram from the upper esophageal short axis view shows a large mass (arrow) that nearly occludes the right pulmonary artery (rPA). There appears to be no mass effect on the main pulmonary artery $(\mathrm{mPA}) . \mathrm{SVC}=$ superior vena cava; Ao $=$ ascending aorta

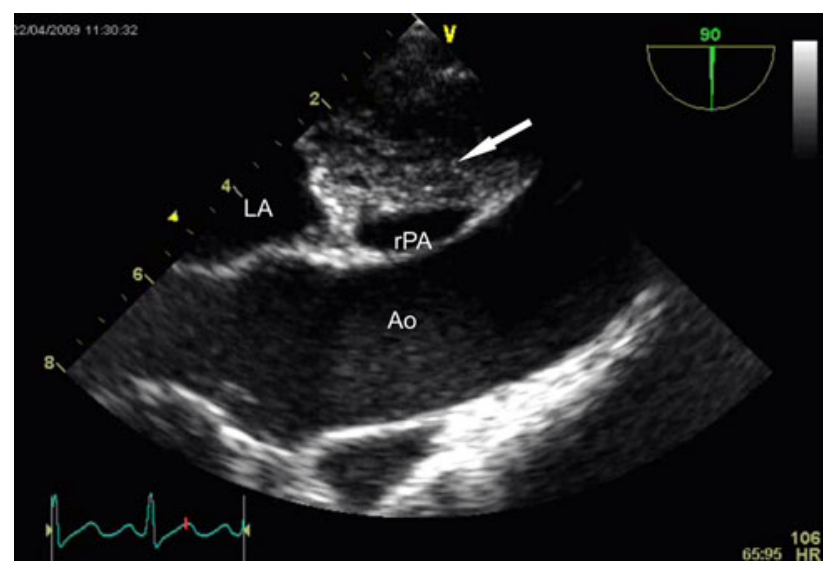

Fig. 2 A transesophageal echocardiogram from the upper esophageal long axis view shows the mass (arrow) compressing the right pulmonary artery (rPA) and abutting the left atrium (LA). Ao = ascending aorta

the procedure. The patient's initial hemodynamic stability with induction and gentle positive pressure ventilation as well as the TEE imaging gave us confidence that hemodynamic collapse was unlikely. The location of the mass with respect to the left PA, main PA, and cardiac chambers suggested that these structures were not at risk of compression. ${ }^{3}$ Masses that compromise the complete pulmonary circulation, cardiac chambers, or superior vena cava are most at risk for hemodynamic decompensation. ${ }^{4}$ As well, given that the right PA was nearly completely occluded without adverse effects, it was reasoned that any further compression of this vessel would be well tolerated.

This case reveals the extent to which simple follicular lymphoid hyperplasia can grow and cause compression of mediastinal structures. In this case, TEE imaging helped to clarify the degree of compression caused by the mass and allowed us to modify our anesthetic technique. Intraoperative TEE is a valuable tool for non-cardiac cases where tumours cause mass effect or compression of the great vessels and cardiac chambers.

Financial support None.

Conflicts of interest None declared.

\section{References}

1. Eltzschig HK, Rosenberger P, Loffler M, Fox JA, Aranki SF, Shernan SK. Impact of intraoperative transesophageal echocardiography on surgical decisions in 12,566 patients undergoing cardiac surgery. Ann Thorac Surg 2008; 85: 845-52.

2. Pullerits $J$, Holzman R. Anaesthesia for patients with mediastinal masses. Can J Anaesth 1989; 36: 681-8.

3. D'Cruz IA, Feghali N, Gross CM. Echocardiographic manifestations of mediastinal masses compressing or encroaching on the heart. Echocardiography 1994; 11: 523-33.

4. Erdos G, Tzanova I. Perioperative anaesthetic management of mediastinal mass in adults. Eur J Anaesthesiol 2009; 26: 627-32. 